\title{
Coding in the olfactory system: linking realistic and abstract models
}

\author{
Christoph Metzner \\ From 1st International Workshop on Odor Spaces \\ Hannover, Germany. 4-7 September 2013
}

In general, theoretical models for the function of the olfactory system can be divided into two broad categories. In one, the seemingly diffuse and unstructured pattern of neuronal connections within the olfactory cortex have been interpreted to suggest that it functions like a kind of an autoassociative neural network $[4,5,7,6]$. An essential feature of autoassociative networks is their randomly structured connectivity and the special importance of the formation of connection weights in the process of its evolution [8,9]. On the other hand, a very different functional structure has recently been proposed based on a detailed biologically realistic network model of the olfactory cortex [11]. This work predicts that the olfactory cortex actually has a highly structured connectivity consisting of distinct different although highly spatially overlapping subnetworks, which are only sparsely connected to each other [11].

In a new collaboration (O'Connor and Steuber,University of Hertfordshire; Bower, University of Texas Health Science Center San Antonio; Metzner, University of Lübeck), we focus on linking models of the olfactory cortex [11] and the olfactory bulb [10] in order to test both the associative and subnetwork theories of olfactory coding.

In this presentation we will focus on a more abstract model, derived from the realistic model in order to apply information theoretic techniques to determine the coding potential of both forms of information representation. This new abstract fused model will follow along the lines of a previous effort to construct a more abstract representation of the original Wilson olfactory cortical model by Crook et al. $[3,2,1]$. In this approach interaction functions for a coupled oscillator model are derived from a biophysically detailed model accurately reflecting the properties of this model. The fact that the interaction

\footnotetext{
Institute for Robotics and Cognitive Systems - University of Luebeck,
} Luebeck, Germany

functions are tightly linked to the experimentally grounded biophysical model, rather than chosen arbitrarily, enables this approach to make accurate predictions about the dynamical behaviour of the whole system and to apply new methods of mathematical and information theoretical analysis to more quantitatively compare the two different theories of olfactory cortical structure and function.

Published: 16 April 2014

\section{References}

. Crook SM, Ermentrout GB, Bower JM: Dendritic and synaptic effects in systems of coupled cortical oscillators. J Comput Neurosci 1998, 5:315-29.

2. Crook SM, Ermentrout GB, Bower JM: Spike frequency adaptation affects the synchronization properties of networks of cortical oscillations. Neural Comput 1998, 10:837-54.

3. Crook SM, Ermentrout GB, Vanier MC, Bower JM: The role of axonal delay in the synchronization of networks of coupled cortical oscillators. J Comput Neurosci 1997, 4:161-72.

4. Haberly LB: Neuronal circuitry in olfactory cortex: anatomy and functional implications. 1985, 10:219-238.

5. Haberly LB, Bower JM: Olfactory cortex: model circuit for study of associative memory? Trends Neurosci 1989, 12:258-64.

6. Barkai $\mathrm{E}, \mathrm{Hasselmo} \mathrm{MH}$ : Acetylcholine and associative memory in the piriform cortex. Mol Neurobiol 1997, 15:17-29.

7. Hasselmo ME, Wilson MA, Anderson BP, Bower JM: Associative memory function in piriform (olfactory) cortex: computational modeling and neuropharmacology. Cold Spring Harb Symp Quant Biol 1990, 55:599-610.

8. Hopfield J: Neural networks and physical systems with emergent collective computational abilities. Proc Natl Acad Sci U S A 1982, 79:2554-2558.

9. Hopfield J: Neurons with graded response have collective computational properties like those of two-state neurons. Proc Natl Acad Sci U S A 1984, 81:3088-3092.

10. O'Connor S, Angelo K, Jacob TJC: Burst firing versus synchrony in a gap junction connected olfactory bulb mitral cell network model. Front Comput Neurosci 2012, 6:75.

1. M Vanier: Realistic computer modeling of the mammalian olfactory cortex. PhD thesis California Institute of Technology; 2001.

doi:10.1186/2044-7248-3-S1-P10

Cite this article as: Metzner: Coding in the olfactory system: linking realistic and abstract models. Flavour 2014 3(Suppl 1):P10. 